\title{
UTILISATION DE DIFFÉRENTS PRODUITS ODORANTS DE SYNTHESE POUR FACILITER LA DÉTECTION DES CHALEURS CHEZ LA TRUIE
}

\author{
J. P. SIGNORET et J. BARITEAU* \\ Station de Physiologie de la Reproduction, \\ Centre de Recherches de Tours, I. N.R. A., \\ Nouzilly, 37380 Monnaie \\ * Station Expérimentale d'Insémination artificielle \\ 86480 Rouille (France)
}

\section{RÉSUMÉ}

L'efficacité de divers dérivés odorants pour faciliter l'apparition de la réaction caractéristique d'immobilisation de la truie en œstrus a été mise à l'épreuve dans les conditions pratiques de l'insémination artificielle. Parmi les 5 produits mis à l'essai, trois ont une action comparable à celle de l'odeur du verrat. De plus, le taux de gestation obtenu après insémination des truies dont l'immobilité a été ainsi induite est nettement amélioré.

\section{INTRODUCTION}

Le bilan économique de l'élevage est conditionné par la fertilité et la prolificité : ainsi, il a été calculé qu'un retard d'un cycle équivaut à la perte d'un porcelet. (CaUgant, I973) On conçoit l'importance que revêt dans ces conditions une bonne détection de 1'œstrus.

Pour cela, la présentation quotidienne des truies à un mâle est la méthode la plus précise, mais les problèmes posés par la manipulation journalière des animaux, et surtout le temps nécessaire à une telle opération limitent l'emploi de cette technique aux stations expérimentales. Il est donc nécessaire de se fier à l'observation directe par l'éleveur. De nombreuses modifications anatomiques (coloration et gonflement de la vulve, mucus, etc.) ou comportementales, accompagnent les chaleurs chez la truie, mais le seul critère fiable est l'apparition d'une réaction d'immobilisation lorsque la truie est chevauchée. Cependant en l'absence du verrat un pourcentage 
important des truies en chaleur ne présentent pas cette réaction caractéristique. Ce fait en limite l'intérêt pour la détermination du moment de l'insémination. Toutefois, nous avons montré (Signore'r et du Mesnil du Buisson, ig6I) que les émissions sonores et odorantes du mâle pouvaient faciliter l'apparition de ce réflexe dans la plus grande partie des cas. L'odeur active est notamment présente dans la sécrétion préputiale du mâle.

L,e produit responsable de cette action a été isolé par PATTERSON (I968) : il s'agit d'un dérivé de la testostérone, le 5-alpha-androsta-I6-en-3-one. Ce produit formé par les glandes salivaires du mâle, diffuse dans les graisses auxquelles il communique une odeur désagréable caractéristique de la viande de verrat. Ce composé présenté sous la forme d'un aérosol, a été utilisé avec succès par MeLrose et al. (I97I) pour améliorer la détection de l'œestrus. Divers autres dérivés voisins se sont révélés également efficaces. (REED et al., I974).

Nous avons, par ailleurs, montré que les truies immobiles lors de l'insémination artificielle, sont fécondées dans une proportion beaucoup plus importante que celles qui sont négatives, puisque la différence des taux de mise bas varie entre 20 et 25 points de pourcentage (du Mesnil du Buisson et Signoret, 1966).

Nous avons voulu mettre à l'essai l'utilisation de l'odeur sexuelle dans les conditions de la pratique de l'insémination artificielle dans le but de préciser si, outre l'avantage de faciliter la détection de l'œestrus, le fait d'induire l'immobilisation des truies par un stimulus odorant améliorait ou non leur taux de fécondation. Nous avons en outre procédé à l'essai d'un certain nombre de composés nouveaux de formule voisine de celui mis en évidence par PATTERson (I968) (1).

\section{MATÉRIEL ET MÉTHODES}

Les essais se sont déroulés au centre expérimental d'insémination de l'I. N. R. A. à Rouillé (Vienne). Les produits, conditionnés en "bombes " permettant d’obtenir un aérosol, ont été utilisés par les inséminateurs au cours des inséminations normales du centre entre février et avril I974. Une semaine sur deux un inséminateur à utilisé un produit odorant, l'autre semaine, il se contentait de la mise en évidence de la présence ou de l'absence de la réaction d'immobilisation. Les différents produits mis à l'épreuve ont été sucessivement utilisés par les divers inséminateurs.

Lors de l'essai, l'inséminateur met d'abord à l'épreuve la réaction de la truie au chevauchement. Si celle-ci est négative, il vaporise pendant 3 à 5 secondes un aérosol de l'un des produits dans la direction des narines de l'animal en tenant le récipient à une cinquantaine de centimètres de la truie. Il éprouve à nouveau la réaction d'immobilisation. L'insémination est alors effectuée selon les techniques de routine du centre.

\section{RÉSULTATS ET DISCUSSION}

Les différents composés mis à l'épreuve se sont révélés d'une efficacité variable : les taux d'immobilisation obtenus (tab1. I) changent selon les produits. Pour deux d'entre eux, ils ne sont pas différents de ce que l'on obtient avec la sécrétion prépu-

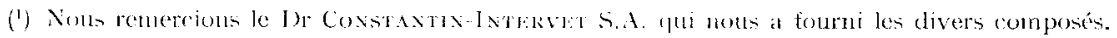


tiale du verrat, ou avec les produits isolés par PATTERSON (I968). Le troisième produit n'est que légèrement inférieur tandis que pour les deux derniers, les taux de réactions enregistrées sont faibles.

\section{TABIEAU I}

Effcacité compavée de différents produits odorants sur l'immobilisation des truies proposées à l'insémination artificielle

\begin{tabular}{|c|c|c|c|}
\hline Produit & $\begin{array}{l}\text { Nombre de } \\
\text { truies négatives }\end{array}$ & $\begin{array}{c}\text { Nombre de } \\
\text { truies immobilisées }\end{array}$ & $\begin{array}{l}\text { Taux d'immobilisation } \\
(\%)\end{array}$ \\
\hline $\begin{array}{l}\text { Sécrétion préputiale } \\
\text { (Signoret et du MeSNIL, 1961) }\end{array}$ & 80 & 42 & $52,5(1)$ \\
\hline $\begin{array}{l}5 \text { alpha-androsta-16-ène-3-one } \\
\text { (MELrose et al., 1971) }\end{array}$ & 57 & 30 & $52,6(1)$ \\
\hline $\begin{array}{c}3 \text { alpha-hydroxy-5 alpha-androsta-16-ène } \\
\text { (NELrose et al., 1971) }\end{array}$ & 32 & 14 & $43,7(2)$ \\
\hline 5 alpha-estra-16-3 alpha-el L (2292) & 197 & 123 & $62,5(1)$ \\
\hline 5 alpha-estra-1 $6-3$ bêta-el (H 2468) & 176 & 50 & $28,4(3)$ \\
\hline 5 alpha-estra-16-3-one (I 2253) & 237 & 98 & $41,4(2)$ \\
\hline 5 alpha-androsté-16-3 bêta-el (JNF 55$)$ & 135 & 69 & $51,1(1)$ \\
\hline $\begin{array}{l}17 \text { méthyl-18 nor-5 alpha- } \\
\text { androsta-13(17)-ène-3-one }(6228 \text { Wo })\end{array}$ & 132 & 30 & $22,7(3)$ \\
\hline
\end{tabular}

Les valeurs suivies du même signe 1-2 ou 3 ne diffèrent pas significativement entre elles.

Les valeurs notées (1) ou (2) sont significativement différentes de celles notées (3) au niveau 1 p. 100. 5 p. 100.

Les différences entre les valeurs notées (1) et (2) sont à la limite de la signification statistique au niveau

Les taux de gestation obtenus chez les truies inséminées après immobilité, induite par l'un ou l'autre des composés odorants, ne sont pas significativement différents (tabl. 2). Ils paraissent donc sans rapport avec l'efficacité de ce produit pour induire 1'immobilité.

Les réactions physiologiques associées à la réaction d'immobilisation apparaissent nécessaires à l'obtention d'un taux de gestation élevé. Enn effet, les truies dont l'immobilisation a été induite par l'effet d'un produit odorant sont gestantes dans une proportion analogue à celles dont la réaction était apparue spontanément $(65,9 \mathrm{p}$. Ioo contre $67,3 \mathrm{p}$. Ioo et $69,4 \mathrm{p}$. I00). Au contraire celles qui demeurent négatives conservent le bas niveau de fécondation qui est associé à ce type de réponse (44, I p. Ioo et 46,4 p. Ioo) (tabl. 3).

Ces essais ont permis de mettre en évidence des différences dans l'efficacité des divers composés odorants mis à l'épreuve. Les plus actifs possèdent une action comparable à celle de la substance sécrétée par le mâle. 
TABLEAU 2

Résultats de fécondation obtenus chez des truies dont la réaction d'immobilisation a été facilitée par l'emploi de divers composés odorants

\begin{tabular}{c|c|c|c|c}
\hline $\begin{array}{c}\text { Composé } \\
\text { odorant utilisé }\end{array}$ & $\begin{array}{c}\text { Nbre de truies } \\
\text { immobilisées }\end{array}$ & $\begin{array}{c}\text { Nbre de } \\
\text { mise bas }\end{array}$ & $\begin{array}{c}\text { Pourcentage } \\
\text { de mise bas }\end{array}$ & $\begin{array}{c}\text { Taille } \\
\text { de la porté }\end{array}$ \\
\hline L 2292 & 123 & 88 & 71,5 & 10,7 \\
\hline H 2268 & 50 & 29 & 58,0 & 10,6 \\
\hline I 2253 & 98 & 55 & 56,1 & 10,0 \\
\hline JNF 55 & 69 & 47 & 68,1 & 10,0 \\
\hline 6228 WO & 30 & 25 & 83,8 & 9,1 \\
\hline \hline
\end{tabular}

TABLEAU 3

Résultats de fécondation obtenus chez des truies inséminées artificiellement en fonction de la réaction d'immobilisation spontanée ou facilitée par l'emploi de produits odorants

\begin{tabular}{c|c|c|c|}
\hline $\begin{array}{c}\text { Réaction } \\
\text { de la Truie }\end{array}$ & $\begin{array}{c}\text { Nombre } \\
\text { I.A. }\end{array}$ & $\begin{array}{c}\text { Nombre } \\
\text { M.B. }\end{array}$ & $\begin{array}{c}\text { M.B. } \\
(\%)\end{array}$ \\
\hline
\end{tabular}

Aver essai des produits odorants

\begin{tabular}{c|c|c|c|c}
\hline $\begin{array}{c}\text { Immobilité } \\
\text { immédiate }\end{array}$ & 1907 & 1284 & 67,3 & 10,3 \\
\hline $\begin{array}{c}\text { Immobilité } \\
\text { induite après odeur }\end{array}$ & 370 & $2 \%$ & 65,9 & 10,2 \\
\hline $\begin{array}{c}\text { Non immobiles } \\
\text { après odeur }\end{array}$ & 127 & 56 & 44,1 \\
\hline C.
\end{tabular}

Sans essai de produits odorants

\begin{tabular}{c|c|c|c|c}
\hline $\begin{array}{c}\text { Immobilité } \\
\text { immédiate }\end{array}$ & 1807 & 1254 & 69,4 & 10,3 \\
\hline Non immobile & 541 & 251 & 46,4 & 9,7 \\
\hline
\end{tabular}


L'emploi de tels produits permet d'améliorer la précision de la détection des chaleurs en facilitant l'apparition de la réaction d'immobilisation en l'absence du verrat.

En outre, et surtout, l'induction de l'immobilisation permet d'accroitre de 20 points le pourcentage le taux de gestation chez les truies qui réagissent à cette stimulation.

L'utilisation de composés odorants pour faciliter l'apparition de l'immobilisation n'est done pas seulement une aide efficace pour détecter les chaleurs, mais constitue par là un moyen d'élever le taux de fécondation obtenu par insémination artificielle chez les truies qui n'étaient pas spontanément immobiles.

Reçu pour publication en mai 1975.

\section{SUMMARY}

UTILIZATION OF VARIOUS SYNTHETIC ODOROUS PRODUCTS

TO FACILITATE DETECTION OF HEATS IN THE SOW

The action of 5 odorous steroids to facilitate the standing reaction of oestrous sows presented for insemination was investigated. Three of them were as effective as the natural 5-alphaandrost-I6-en-3-one and the boar's preputial secretion. The fertility of sows inseminated after a positive response to the treatment was significantly improved $\left(244 / 37^{\circ}=65.9 \mathrm{p}\right.$. Ioo) when compared to non standing untreated sows (25I/54I $=46.4 \mathrm{p}$. Ioo) and non standing treated sows $(56 / 127=44.1$ p. IOo).

\section{RÉFÉRENCES BIBLIOGRAPHIQUES}

Caugant A., 1973. Combien coûtent nos mauvaises performances? Bull. I. T. P., r9-24.

Melrose D. R., Reed H. C. B., Patterson R. L. S., r97I. Androgen steroids associated with boar odor as an aid to the detection of oestrus in pig artificial insemination. Brit. Vet. J., 127, 497-502.

Du Mesnil du Bursson F., Signoret J. P., I966. Facteurs femelles affectant la fertilité dans la pratique de l'insémination porcine. World Rev. Anim. Prod., 2, 45-54,

Patterson R. L. S., I968. 5 alpha-androst-I6-en-3-one : compound responsible for taint in boar fat. J. Sci. Fd. A gric., 19, $3 \mathrm{I}-38$.

Reed H. C. B., Melrose D. R., Patterson R. L. S., 1974. Androgen steroids an aid to the detection of oestrus in pis artificial insemination. Brit. Vet. J., 130, 6r-67.

Signoret J. P., Du Mesnil, du Buisson F., ig6r. Étude du comportement de la Truie en oestrus. IVe Cong. intern. Reprod. anim., La Haye, I7I-I75. 